\title{
Muss es bei Diabetes immer ein RAS-Blocker sein?
}

\author{
Die Präferenz für RAS-Blocker bei Hypertonie und Diabetes mellitus ist ein kaum infrage \\ gestellter Brauch. Nun kommt eine Metaanalyse zu überraschenden Ergebnissen.
}

_ Für die Metaanalyse wurden die einschlägigen Datenbanken nach randomisierten Studien durchsucht, in denen Hemmstoffe des Renin-AngiotensinSystems (RAS) gegen andere Antihypertensiva bei Diabetikern getestet wurden. Endpunkte waren Tod, Tod mit kardiovaskulärer Ursache, Herzinfarkt, Angina pectoris, Schlaganfall, Herzinsuffizienz, Revaskularisierung und terminale Niereninsuffizienz. 19 randomisierte, kontrollierte Studien mit 25.414 Diabetikern und 95.910 Patientenjahren wurden ausgewertet.

Für keinen der Endpunkte ergab sich ein signifikanter Unterschied zwischen RAS-Blockern und Antihypertensiva wie Thiaziden, Kalziumkanal- oder Betablockern.

- Bangalore Set al. Diabetes mellitus as a compelling indica tion for use of renin angiotensin system blockers: systematic review and meta-analysis of randomized trials. BMJ. 2016;352:i438

\section{KOMMENTAR}

Bei Diabetikern sind RAS-Blocker der Analyse zufolge anderen Antihypertensiva hinsichtlich der Senkung harter Endpunkte nicht überlegen. Entscheidend ist offenbar vor allem die effektive Blutdrucksenkung. Gerade hier sind nach SPRINT und Metaanalysen, die zu ähnlichen Ergebnissen kamen, bald Leitlinienänderungen zu erwarten. Die Wahl des Antihypertensivums scheint hingegen nicht entscheidend zu sein.

Es ist allerdings darauf hinzuweisen, dass diese Schlussfolgerungen nicht für Diabetiker mit Niereninsuffizienz gelten. Für diese Patientengruppe ist auch in Anbetracht dieser Studie der RAS-Blocker das bevorzugte Antihypertensivum. Für die Diabetiker mit Nierenerkrankung lassen sich aus der vorliegenden Metaanalyse keine verlässlichen Aussagen machen. Die Charakterisierung der Nephropathie war in den einzelnen Studien, die Eingang in die Analyse gefunden haben, zu unterschiedlich, um die Schlussfolgerung auch auf diese Subgruppe anzuwenden. Von den verschiedenen aktuellen Leitlinien sind lediglich jene des Eighth Joint National Committee (JNC8) auf der Linie der Metaanalyse, da sie für Diabetiker ohne Nephropathie keine Präferenz zugunsten der RASBlocker aussprechen. Salomonisch, aber mit einer deutlichen Empfehlung zugunsten der RAS-Blocker äußern sich die europäischen ESH-Leitlinien (RAS-Blockern sollte "der Vorzug gegeben werden") wie auch die amerikanischen/internationalen ASH/ISH-Leitlinien (RAS-Blocker "sollten verwendet werden, speziell bei Mikroalbuminurie oder Nierenerkrankung").

Glücklicherweise ist die Entscheidung in der Praxis nicht allzu schwierig, da die meisten hypertensiven Diabetiker ohnehin eine Kombinationstherapie benötigen, die in der Regel auch einen RAS-Blocker enthält.

Prof. Dr. med. W. Zidek

\section{Intrafaziale Synkinesie}

Ein 52-jähriger Mann wurde zur Abklärung einer seit Wochen bestehenden allgemeinen Muskelschwäche stationär aufgenommen. Bei der Untersuchung der Hirnnerven fiel auf, dass es bei der willkürlichen Kontraktion der unteren Gesichtsmuskeln zu einer unwillkürlichen linksseitigen Ptose kam. Diese Form der Synkinesie ist beschrieben als Marin-Amat-Syndrom. Es bestand eine symmetrische proximale Muskelschwäche. In Zusammenschau von Gottron-Papeln an der Streckseite der Fingermittelgelenke und einer massiv erhöhten Kreatinkinase von $51.000 \mathrm{U} / \mathrm{I}$ wurde die Diagnose einer Dermatomyositis gestellt. Die Synkinesie war allerdings mit dieser Diagnose nicht vereinbar. Bei erneutem Befragen berichtete der Patient über eine linksseitige Fazialisparese, die vor einigen Jahren aufgetreten war. Offensichtlich lebte er mit diesem Restsymptom schon mehrere Jahre lang, ohne dass es ihn störte.

Unter einer Synkinesie versteht man die unwillkürliche Mitbewegung eines Muskels bei der Bewegung eines anderen. Das Marin-Amat-Syndrom, eine spezifische Form der orofazialen Synkinesie, beschreibt die Kontraktion des M. orbicularis oculi bei der willkürlichen Bewegung der unteren Gesichtsmuskulatur. Sie entsteht hauptsächlich durch eine aberrierende Regeneration von Nervenfasern nach Verletzungen, kann sich aber auch nach einer Fazialisparese als Defektheilung ausbilden. Die Be-

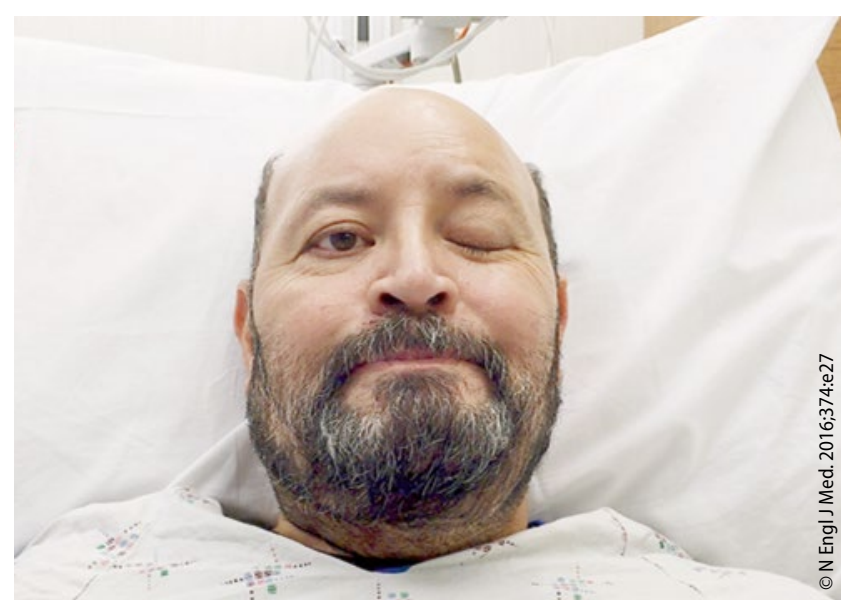

Linksseitige Ptose nach Kontraktion der Gesichtsmuskeln.

handlungsmöglichkeiten bestehen in einem neuromuskulären Training der Gesichtsmuskulatur oder der Injektion von Botulinumtoxin.

Prof. Dr. med. H. S. FüeßI

- Mansoor M, Sullivan PD (mansooan@ohsu.edu). Intrafacial sykinesis. N Engl J Med. 2016;374:e27 